\title{
Derivation of A New Bioscore for Predicting Mortality
} in Sepsis

Shukeri WFWM ${ }^{a, b}$, Md-Ralib $A^{a}$, Mat-Nor MB ${ }^{a^{*}}$

a Department of Anaesthesiology and Intensive Care, Kulliyyah of Medicine, International Islamic University

Malaysia, 25200 Kuantan, Pahang, Malaysia

Department of Anaesthesiology and Intensive Care, School of Medical Sciences, Universiti Sains Malaysia, 16150 Kubang Kerian, Kelantan, Malaysia

\section{ABSTRACT}

Introduction: Currently, there is a lack of clinically feasible and reliable method for discriminating outcome in sepsis. We aimed to derive a new bioscore for predicting mortality in critically ill patients with sepsis using a combination of biomarkers and clinical indexes. Materials and Methods: This was a secondary analysis from a prospective study involving 159 patients with sepsis admitted to an intensive care unit (ICU). Data for key variables considered for possible inclusion in the score were collected, which included: age, sex, source of admission, comorbidities, microorganism, bacteraemia, site of infection, septic shock status, baseline Simplified Acute Physiological Score II, Sequential Organ Failure Assessment (SOFA) score (total and organ sub-scores), C-reactive protein, procalcitonin and interleukin-6 (IL-6). Approximate quintiles of each variable were given points as per the strength of their association with 30-day mortality. Results: In accordance with the statistical significance in the logistic regression analysis, the final score utilised candidate variables of age, central nervous system and liver SOFA sub-scores and IL-6. The bioscore predicted 30-day mortality with a very good performance [area under the receiver operating characteristic curve 0.814 ( $95 \%$ confidence interval $0.745-0.871, p<0.0001)$ ] in our sepsis cohort. A bioscore greater than 4 predicted 30 -day mortality with $80.4 \%$ sensitivity, $69.9 \%$ specificity, 2.67 positive likelihood ratio and 0.28 negative likelihood ratio. As the score increased, so did mortality rate. Conclusion: A new bioscore combining age, central nervous system and liver SOFA sub-scores and IL-6 measured on ICU admission potentially improves prediction of mortality in sepsis. Further study is warranted to prospectively validate the clinical utility of this bioscore in risk-stratifying patients with suspected sepsis.

KEYWORDS: sepsis; biomarkers; critical care; mortality.

\section{INTRODUCTION}

Once sepsis is diagnosed, prediction of survival should be performed. This is remarkably important because high-risk patients may benefit the most from aggressive medical care, while low-risk patients may benefit from not undertaking unnecessary interventions. ${ }^{1}$ Thus, knowing where the patients reside on the spectrum of sepsis may lead to improved outcomes. However, accurate

Corresponding Author

Assoc. Prof. Dato' Dr. Mohd Basri Mat-Nor, Consultant Intensivist and Associate Professor, Department of Anaesthesiology and Intensive Care, Kulliyyah of Medicine, International Islamic University Malaysia, 25200 Kuantan, Pahang, Malaysia.

Tel : +6095912610

E-mail: basri.matnor@gmail.com evaluation of septic patients who are at risk of mortality remains challenging. In recent years, various biomarkers have been investigated for prognostication of sepsis. Nevertheless, owing to its complex pathophysiology, no single biomarker could reliably predict the outcome of sepsis. ${ }^{2}$ A few studies reported the combined use of biomarkers could improve outcome prediction in sepsis. ${ }^{3-6}$ Nevertheless, this approach may be expensive and not feasible to be routinely employed in clinical practice. Therefore, it is of clinical relevance to derive a simpler scoring system for discriminating outcome in sepsis.

In the Third International Consensus Conference for Sepsis and Septic Shock (Sepsis-3), sepsis has recently been defined as dysregulated host response to infection causing organ dysfunction. ${ }^{7}$ In Figure 1, 
in line with this definition, we presented our conceptual model on how the host factors, the response factors, the infection factors and the organ dysfunction factors in sepsis eventually impact the patient outcomes. Our eventual goal was to derive a score from the variables shown in the conceptual model that would estimate the likelihood of mortality in an individual patient with sepsis. Thus, in the present study, we aimed to develop a scoring system, or a bioscore, for predicting 30-day mortality in critically ill patients with sepsis using combination of biomarkers and clinical indexes.

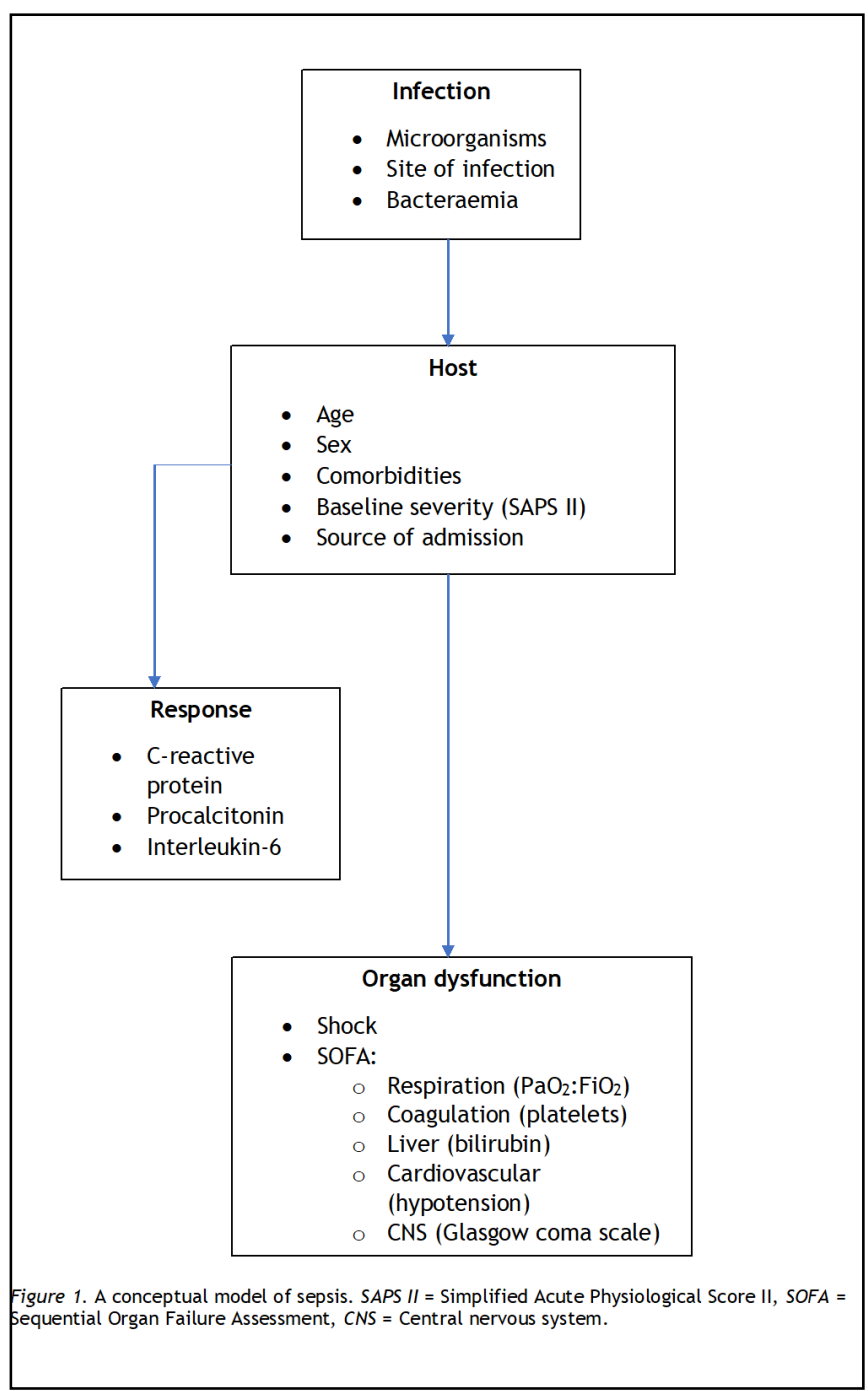

\section{MATERIALS AND METHODS}

\section{Study Designs and Participants}

Secondary analysis was conducted using data from a prospective cohort study performed in the intensive care unit (ICU) of a tertiary hospital referral centre in Pahang, Malaysia, over a three-year period (from July 2011 to June 2014). After consent was obtained, consecutive adult patients diagnosed with Systemic Inflammatory Response Syndrome (SIRS) were enrolled into the study. The exclusion criterion was administration of antimicrobial therapy for greater than 24 hours before baseline blood samples for biomarker analysis were taken. The original purpose of this investigation was to assess the diagnostic and prognostic utilities of several biomarkers and has been previously reported. ${ }^{8}$ The study received ethical approval from the local institutional research ethics committee and included consent for secondary analysis of the data collected. The study protocol was registered under the National Medical Research Registry (NMRR-13-1592-18706). In this study, we carried out a secondary analysis to derive a bioscore from the data collected for predicting 30-day mortality in sepsis.

Initially, 239 patients diagnosed with SIRS were recruited. For this analysis, we excluded 80 patients with no suspected infection and not fulfilling the Sepsis-3 criteria. Thus, 159 patients were finally included in the analysis (Figure 2). This sample size satisfied the requirement that a minimum of 80 patients were needed to attain the relevant anticipated area under the receiver operating characteristic curve (AUROC) of 0.7 at a $5 \%$ significance level, $90 \%$ power and $20 \%$ dropout rate.

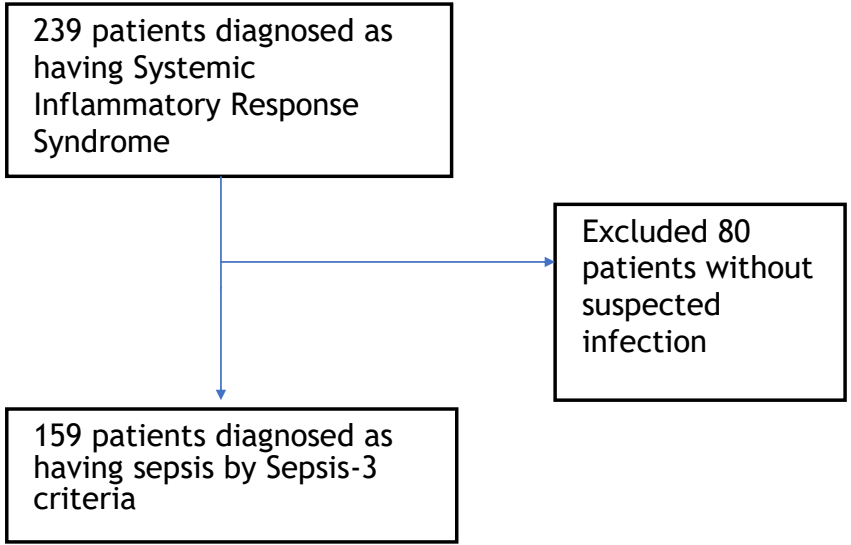

Figure 2. A flow chart for patient recruitment. SOFA = Sequential Organ Failure Assessment

For the 159 patients enrolled, pertinent demographic and clinical data were recorded, including age, sex, comorbidities as assessed by Charlson Comorbidity Index, baseline severity as represented by Simplified Acute Physiological Score (SAPS) II, source of admission, microorganisms, site of infection, presence of bacteraemia, septic shock status, baseline Sequential Organ Failure Assessment (SOFA) score in terms of both its organ sub-scores and total score, and baseline biomarkers of Creactive protein (CRP), procalcitonin (PCT) and 
interleukin-6 (IL-6). Patients were followed-up to assess for 30-day mortality.

\section{Assays}

Within 24 hours after ICU admission, blood sampling for measurement of CRP, PCT and IL- 6 was done. Measurement of serum CRP concentrations was achieved using a turbidimetric immunoassay test (BNII, Siemens Healthcare Diagnostic, Germany). Serum PCT concentrations were measured based on time-resolved amplified cryptate emission technology, by the BRAHMS Kryptor compact assay (Henningsdorf, Germany). Lastly, serum IL-6 concentrations were quantified using the Quantikine enzyme-linked immunosorbent assay kit provided by R\&D Systems (Minnesota, USA).

\section{Statistical Analysis}

All statistical analysis was conducted with MedCalc for Windows, version 17.5.5 (MedCalc Software, Ostend, Belgium) and SPSS version 24.0 (IBM, Armonk, NY, USA). Our first step was to describe the association between all the variables with 30 day mortality, thus validating our choice of candidate variables in the conceptual model. This was done by comparing candidate variables between 30-day survivors and non-survivors. Categorical variables were presented as frequencies and percentages and compared by the Chi-Square test whereas continuous variables were presented as mean \pm standard deviation or median (interquartile range) and compared by the independent $\mathrm{t}$ test or the Mann-Whitney. For quantitative variables, Kolmogorov-Smirnov test $(P>0.10)$ was used to test for normality of distribution.

In the second step, we constructed the bioscore using the candidate predictor variables. Each SOFA parameters were left as integer values (range 0 to $5)$, while the rest of the candidate variables were divided into quintiles. Each of the variables were then fit as categorical predictors in separate binary logistic regression, with 30 -day mortality status as the outcome. The parameters for each logistic model estimate the logit for each category of the variable compared with the lowest risk (reference) category. These parameters were rounded to the nearest whole numbers to come up with the points used in the bioscore. Categories with similar points were collapsed, and the exact quintile ranges were then rounded to convenient numbers. The full bioscore was straightforwardly the total of the points across all included variables. Variables with a P-value of greater than 0.2 or with all categories allocated 0 points were denied from the score. Additionally, variables were excluded if their inclusion in the bioscore did not enhance the score's capability to prognosticate 30 -day mortality.

In our final step we evaluated the quality of the bioscore in predicting 30-day mortality. The model calibration was assessed by the Hosmer-Lemeshow goodness-of-fit test, while the discrimination was evaluated by the AUROC of the sensitivity (true positive rate) against 1 -specificity (false positive rate) across a series of cut-off points. The AUROC ranges from 0.5 (no discrimination) to 1 (perfect discrimination). The model was assumed to be clinically valid at an AUROC of more than 0.70. The Youden index was applied to set the optimal cut-off point. Sensitivity, specificity and likelihood ratio (LR) of the bioscore for predicting 30-day mortality were calculated for the cut-off point. The differences in the AUROCs of the bioscore and each of its components were evaluated for significance using the DeLong test. ${ }^{9}$ All patients were then dichotomised based on the ideal cut-off value of the bioscore, and the two groups were compared in terms of 30-day mortality by Kaplan-Meier survival curves. A log rank test was carried out to compare the survival curves of the groups.

\section{RESULTS}

\section{Baseline Data}

The characteristics of the whole study population and comparison of candidate variables by 30 -day survivor status are summarized in Table 1. Of note, the outcome of 30-day mortality was reached in 46 (28.9\%) of patients. Candidate predictors significantly associated with 30-day mortality were older age, septic shock, higher SAPS II, higher total SOFA score and all the sub-organ scores except for respiratory and cardiovascular systems, higher Charlson comorbidity index and isolation of gram negative organism (all $p<0.05$ ). All the studied biomarkers namely CRP, PCT and IL-6 were significantly associated with 30-day mortality (all $p$ $<0.05)$. 
Table 1. Whole population characteristics and difference between 30 -day survivors and non-survivors.

\begin{tabular}{|c|c|c|c|c|}
\hline Variables & $\begin{array}{l}\text { All } \\
(n=159)\end{array}$ & $\begin{array}{l}\text { Survivors } \\
(n=113)\end{array}$ & $\begin{array}{l}\text { Non-survivors } \\
(n=46)\end{array}$ & $P$ \\
\hline Age (years) & $47 \pm 16$ & $45 \pm 16$ & $54 \pm 16$ & 0.002 \\
\hline Sex (male) & $109(68.6)$ & $73(64.6)$ & $36(78.3)$ & 0.093 \\
\hline $\begin{array}{l}\text { Admission category: } \\
\text { Medical } \\
\text { Surgical }\end{array}$ & $\begin{array}{l}117(73.6) \\
42(26.4)\end{array}$ & $\begin{array}{l}85(75.2) \\
28(24.8)\end{array}$ & $\begin{array}{l}32(69.6) \\
14(30.4)\end{array}$ & $\begin{array}{l}0.463 \\
0.463\end{array}$ \\
\hline $\begin{array}{l}\text { Site of infection: } \\
\text { Lungs } \\
\text { Abdomen } \\
\text { Soft tissue } \\
\text { Urinary tract } \\
\text { Nervous system }\end{array}$ & $\begin{array}{l}90(56.6) \\
15(9.4) \\
13(8.2) \\
9(5.7) \\
8(5.1)\end{array}$ & $\begin{array}{l}65(57.5) \\
10(8.8) \\
11(9.7) \\
7(6.2) \\
8(7.1)\end{array}$ & $\begin{array}{l}25(54.3) \\
5(10.9) \\
2(4.3) \\
2(4.3) \\
0(0)\end{array}$ & $\begin{array}{l}0.714 \\
0.693 \\
0.261 \\
0.648 \\
0.064\end{array}$ \\
\hline Bacteraemia & 27 (16.9) & $16(14.2)$ & $11(23.9)$ & 0.137 \\
\hline $\begin{array}{l}\text { Microorganisms: } \\
\text { Gram positive } \\
\text { Gram negative }\end{array}$ & $\begin{array}{l}13(8.2) \\
14(8.8)\end{array}$ & $\begin{array}{l}10(8.8) \\
6(5.3)\end{array}$ & $\begin{array}{l}3(6.5) \\
8(17.4)\end{array}$ & $\begin{array}{l}0.627 \\
0.015\end{array}$ \\
\hline $\begin{array}{l}\text { Severity of illness: } \\
\text { Septic shock } \\
\text { SAPS II } \\
\text { SOFA: } \\
\text { Respiratory } \\
\text { Coagulation } \\
\text { Liver } \\
\text { CNS } \\
\text { Cardiovascular } \\
\text { Renal }\end{array}$ & $\begin{array}{l}39(24.5) \\
43[33-56] \\
9[6-12] \\
2[1-3] \\
0[0-1] \\
0[0-2] \\
0[0-4] \\
1[0-3] \\
1[0-3]\end{array}$ & $\begin{array}{l}18(15.9) \\
40[30-51] \\
7[5-10] \\
\left.2\left[\begin{array}{c}1-3] \\
0\end{array}\right] 0-1\right] \\
0[0-1] \\
3[0-4] \\
0[0-3] \\
1[0-2]\end{array}$ & $\begin{array}{l}21(45.7) \\
53[41-63] \\
12[9-16] \\
3[1-3] \\
1 \\
1[0-2] \\
4[0-2] \\
1[0-4] \\
2[0-3]\end{array}$ & $\begin{array}{l}<0.0001 \\
<0.0001 \\
<0.0001 \\
0.366 \\
0.002 \\
<0.0001 \\
<0.0001 \\
0.696 \\
0.003\end{array}$ \\
\hline $\begin{array}{l}\text { Charlson } \\
\text { Comorbidity Index }\end{array}$ & $1.8 \pm 2.2$ & $1.4 \pm 1.7$ & $2.6 \pm 3.1$ & 0.019 \\
\hline $\begin{array}{l}\text { C-reactive protein }(\mathrm{mg} / \mathrm{dL}) \\
\text { Procalcitonin }(\mathrm{ng} / \mathrm{mL}) \\
\text { Interleukin-6 }(\mathrm{pg} / \mathrm{mL})\end{array}$ & $\begin{array}{l}17[10-30.1] \\
10.49[1.03-43.07] \\
329.52[87.98-628.89]\end{array}$ & $\begin{array}{l}14.58[10-26.86] \\
8.35[0.95-22.70] \\
154.66[70.67- \\
573.49]\end{array}$ & $\begin{array}{l}26.52[13.02-59.38] \\
22.56[1.62-104.23] \\
491.97[305.63- \\
772.63]\end{array}$ & $\begin{array}{l}0.016 \\
0.009 \\
<0.0001\end{array}$ \\
\hline
\end{tabular}

Note. Data are expressed as mean \pm standard deviation, frequencies (percentage) or median [interquartile range]. The results of the comparison between the two groups was analysed by independent $t$-test, the MannWhitney test for continuous variables or the chi-squared test for categorical variables. SAPS $\|=$ Simplified Acute Physiological Score II, SOFA = Sequential Organ Failure Assessment, CNS = Central nervous system

\section{Derivation of the Bioscore}

In Table 2 we present the variable ranges with their bioscore points used in the final scoring algorithm. Only age, liver SOFA sub-score as represented by serum bilirubin level, central nervous system (CNS) SOFA sub-score as represented by Glasgow coma scale (GCS) and IL-6 were included because the remaining variables were not significantly associated with mortality or their inclusion did not enhance the fit of the final model. The final full scores started from 0 (lowest risk category for all included variables) to 10 (highest risk category for all included variables).

The Hosmer and Lemeshow chi-squared test of the bioscore was 6.213 ( $p>0.05,8$ degrees of freedom), suggesting that the model is well calibrated. When the bioscore was plotted for analysis by receiver operating characteristic curve (Figure 3), the AUROC was 0.814 (95\% confidence interval $0.745-0.871, p$ $<0.0001$ ) which indicated very good model discrimination. The optimal cut-off value of the score was 4. At this cut-off value, the sensitivity and specificity of the bioscore were $80.4 \%$ and $69.9 \%$, respectively, with a positive likelihood ratio of 2.67 and negative likelihood ratio of 0.28 , respectively. The bioscore outperformed its constituent individual variables as well as the SOFA score in predicting the 30-day mortality (Figure 3 and Table 3), with the differences in their AUROCs being statistically significant from the DeLong tests (all $p<0.005$ ).

Table 2. The proposed scoring system for predicting in-hospital mortality in sepsis.

\begin{tabular}{lcc}
\hline Variables & Grading & Range \\
\hline Age & 0 & $<60$ \\
& 1 & $\geq 60$ \\
Serum bilirubin & 0 & $0-19$ \\
level $(\mu \mathrm{mol} / \mathrm{L})$ & 1 & $20-32$ \\
& 2 & $33-101$ \\
& 3 & $102-204$ \\
Glasgow coma scale & 4 & $>204$ \\
& 0 & 15 \\
& 1 & $13-14$ \\
& 2 & $10-12$ \\
Interleukin-6 & 3 & $6-9$ \\
$(\rho g / \mathrm{mL})$ & 4 & $<6$ \\
& 0 & $<400$ \\
& 1 & $\geq 400$ \\
\hline
\end{tabular}


According to the Kaplan-Meier analysis, patients who scored greater with the bioscore had significantly higher mortality rate (log rank test, $p<0.001)$ compared to those with a lower score. The 30-day mortality rate for patients with bioscore more than and less than 4 were $10.2 \% \quad(n=9) \quad$ vs. $52.1 \%$ $(n=37)$, respectively. When patients were classified according to the increasing bioscore, mortality clearly increases with increasing bioscore above its ideal cut-off point in a stepwise fashion: $8.7 \%$ in bioscore $4,30.4 \%$ in bioscore $5,52.2 \%$ in bioscore 6 , $58.3 \%$ in bioscore $7,77.8 \%$ in bioscore 8 and $100 \%$ in bioscore 9 and 10 .

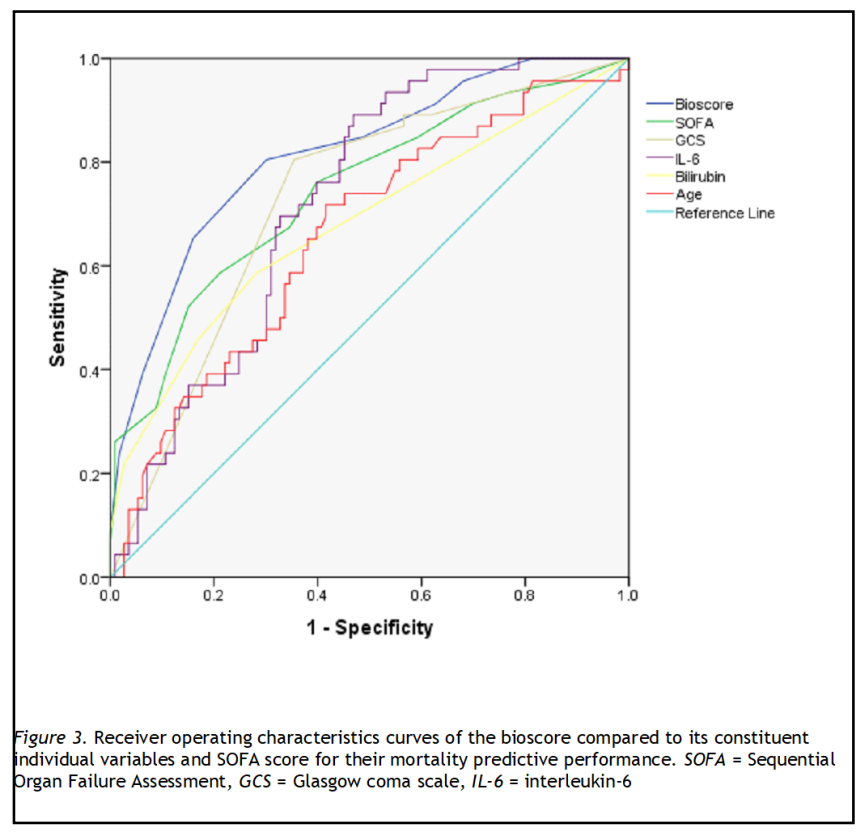

\section{DISCUSSION}

The goal of this secondary analysis of prospectively acquired data was to create a scoring system, or a "bioscore", using combination of biomarker and clinical indexes, that would allow discrimination of critically ill patients with sepsis who are at increased risk of 30-day mortality. A bioscore composed of age, baseline CNS and liver SOFA sub-scores and IL-6 predicted 30-day mortality with a very good performance (AUROC 0.814 ) in our sepsis cohort. At the ideal cut-off point of 4 , the score has $80.4 \%$ sensitivity, $69.9 \%$ specificity, positive LR 2.67 and negative LR 0.28. Of particular note, the bioscore outperformed the prognostic information provided by the SOFA score (AUC 0.744).

The SOFA score, an estimate of multiple organ dysfunction syndrome (MODS), has a widespread use to predict mortality in critically ill patients. ${ }^{10}$ The score assigns uniform ranges of sub-scores, 0 to 4 , to each organ system. ${ }^{11}$ While the uniform weighting of organ failure permits simple calculation, it fails to identify that organ systems may participate unequally to the sequelae of MODS, including mortality. ${ }^{12}$ Our findings demonstrated that combination of CNS and liver SOFA sub-scores, which is determined by GCS and bilirubin, respectively, yielded better prediction of mortality than the total SOFA score.

In one recent study, Knox and colleagues also demonstrated that CNS SOFA sub-score dominated the relationship between admission SOFA score and mortality in critically ill patients. ${ }^{12}$ It is widely recognised that sepsis often is characterised by acute brain dysfunction, which is an independent prognosis factor and associated with increased mortality. ${ }^{13,14}$ Similar to our findings, Patel and colleagues showed that elevated serum bilirubin was an independent predictor of mortality in sepsis. ${ }^{15}$ Increased mortality related to hyperbilirubinemia seem clear and are supported by additional studies. ${ }^{15,16}$

Table 3. Prognostic value of each variable for 30 -day mortality.

\begin{tabular}{|l|c|c|c|c|c|c|}
\hline Variables & AUROC & $P$ value & Sensitivity & Specificity & +LR & -LR \\
\hline Bioscore & 0.814 & $<0.0001$ & 80.43 & 69.91 & 2.67 & 0.28 \\
\hline SOFA & 0.744 & $<0.0001$ & 58.7 & 78.76 & 2.76 & 0.52 \\
\hline GCS & 0.727 & $<0.0001$ & 80.43 & 64.6 & 2.27 & 0.3 \\
\hline Interleukin-6 & 0.724 & $<0.0001$ & 89.13 & 53.1 & 1.9 & 0.2 \\
\hline Bilirubin & 0.681 & $<0.0001$ & 58.7 & 71.68 & 1 & 0.58 \\
\hline Age & 0.660 & 0.0008 & 71.74 & 58.41 & 1.72 & 0.48 \\
\hline
\end{tabular}

$A U R O C=$ Area under the receiver operating characteristic curve, $+L R=$ Positive likelihood ratio, $-L R=$ Negative likelihood ratio, SOFA = Sequential Organ Failure Assessment, GCS = Glasgow coma scale 
Addition of age appears to improve the predictive ability of our bioscore. It is to be expected that age is a consolidate marker of several factors including physiological reserve, comorbidities, magnitude of the medical care desired, and capacity to recover after injury. ${ }^{12}$ In one large study, Martin and colleagues showed that occurrence of sepsis is disproportionately high in elderly adults, and age is an independent predictor of mortality. ${ }^{17}$ In regards to the biomarkers, our result is in line with the work by Fraunberger and colleagues which demonstrated that IL-6 rather than CRP and PCT was an early predictor of mortality in critically ill patients at the first appearance of fever. $.^{18} \mathrm{IL}-6$, a pro-inflammatory cytokine, serves as an important mediator during the acute phase response to inflammation in sepsis. There have been a number of previous studies that strongly supported the ability of early IL-6 levels to predict mortality in sepsis. ${ }^{8,19}$

This study has several pertinent limitations. First, due to the secondary analysis nature of the study, we relied on variables collected as part of the original study to be included in our conceptual model of sepsis and consequently in the bioscore. Some variables were not available but may be important and may increase the prognostic value of the bioscore should they have been included. Examples of these variables include genetic variability, bacterial load and more specific markers of host-response and organ dysfunction to sepsis. ${ }^{20}$ Second, the performance of the bioscore was not externally confirmed in a validation cohort. The predictive pattern may be unique to our cohort but whether it will survive external validation is unknown. Therefore, further research is warranted to validate the clinical utility of the bioscore in an independent cohort. Third, the study cohort is taken from a single ICU staffed by one group of intensivists; thus, the results may not be generalizable to other healthcare settings.

Despite the above-mentioned limitations, our study also has several strengths. Although others have reported that combined use of biomarkers could improve outcome prediction in sepsis, ${ }^{3-6}$ their approach may be expensive and not feasible to be routinely employed in clinical practice, especially in low- and middle-income ICUs. For example, in what is probably the largest study using multi-marker approach in the critical care literature, Shapiro and colleagues utilised a panel of three markers not in common use which are neutrophil gelatinaseassociated lipocalin, protein $\mathrm{C}$ and interleukin-1 receptor antagonists. ${ }^{3}$ We have proposed a simpler scoring system for discriminating outcome in sepsis which may thus be of more clinical relevance. Also, our cohort consisted of a broad patient composition which may increase the generalizability of this study. Furthermore, the data were collected prospectively, which allowed for significantly fewer missing data than in most other secondary analysis studies.

\section{CONCLUSION}

A new bioscore combining age, CNS and liver SOFA sub-scores and IL- 6 measured on admission to the ICU improves prediction of mortality in sepsis. This scoring system may be beneficial in recognizing critically ill patients with sepsis who are at risk of mortality and most likely to benefit from earlier clinical interventions. Further study is warranted to prospectively validate the clinical utility of this bioscore in the risk assessment of critically ill patients with sepsis.

\section{Conflict of Interest: Nil}

\section{ACKNOWLEDGEMENTS}

This work was supported by the International Islamic University Malaysia Research Initiative Grant RIGS 16-113-0277

\section{REFERENCES}

1. Sweeney $T$, Wong $H$. Risk Stratification and Prognosis in Sepsis: What Have We Learned from Microarrays? Clin Chest Med. 2016;37(2):209-18.

2. Reinhart K, Bauer M, Riedemann NC, Hartog CS. New approaches to sepsis: Molecular diagnostics and biomarkers. Clin Microbiol Rev. 2012;25 (4):609-34.

3. Shapiro NI, Trzeciak S, Hollander JE, Birkhahn R, Otero R, Osborn TM, et al. A prospective, multicenter derivation of a biomarker panel to assess risk of organ dysfunction, shock, and death in emergency department patients with suspected sepsis. Crit Care Med. 2009;37(1):96104.

4. Kim H, Hur M, Moon H-W, Yun Y-M, Di Somma S. Multi-marker approach using procalcitonin, 
presepsin, galectin-3, and soluble suppression of tumorigenicity 2 for the prediction of mortality in sepsis. Ann Intensive Care [Internet]. 2017;7(1):27.

5. Dieplinger B, Egger M, Leitner I, Firlinger F, N, Kandelman S, Mantz J, Chrétien F, Sharshar T. Sepsis-induced brain dysfunction. Vol. 11, Expert Review of Anti-Infective Therapy. 2013. p. 211-21.

14. Sonneville R, Verdonk F, Rauturier C, Klein IF, Wolff $M$, Annane D, et al. Understanding brain dysfunction in sepsis. Ann Intensive Care. 2013;3(1):1-11.

15. Patel JJ, Taneja A, Niccum D, Kumar G, Jacobs $E$, Nanchal $R$. The association of serum bilirubin levels on the outcomes of severe sepsis. J Intensive Care Med. 2015;30:23-9.

16. Zhai R, Sheu CC, Su L, Gong MN, Tejera P, Chen $F$, et al. Serum bilirubin levels on ICU admission are associated with ARDS development and mortality in sepsis. Thorax. 2009;64(9):784-90.

17. Martin GS, Mannino DM, Moss M. The effect of age on the development and outcome of adult sepsis. Crit Care Med. 2006;34(1):15-21.

18. Fraunberger $P$, Wang $Y$, Holler $E$, Parhofer KG, Nagel D, Walli AK, et al. Prognostic value of interleukin 6, procalcitonin, and C-reactive protein levels in intensive care unit patients during first increase of fever. Shock. 2006;26 (1):10-2.

19. Jekarl DW, Lee SY, Lee J, Park YJ, Kim Y, Park $\mathrm{JH}$, et al. Procalcitonin as a diagnostic marker and IL- 6 as a prognostic marker for sepsis. Diagn Microbiol Infect Dis. 2013;75(4):342-7.

20. Rabello LSCF, Rosolem MM, Leal J V., Soares M, Lisboa T, Salluh JIF. Understanding the PIRO concept: From theory to clinical practice - Part 1. Rev Bras Ter intensiva. 2009;21(4):425-31. 
